\title{
Measuring the Total Knowledge of English Sports Terminology by the Physical Education Students at Saudi Universities
}

\author{
${ }^{1}$ Majed Saleem El-Saleh, ${ }^{1}$ Abdel Rahman Mitib Altakhaineh*, ${ }^{2}$ Maher Madani \\ Masoudi \\ ${ }^{1}$ Al Ain University of Science and Technology, College of Education, Humanities and Social Sciences, \\ Al Ain, UAE. ${ }^{2}$ College of Education, Taibah University, Medina, KSA.
}

Submitted 29 Mar 2019; Accepted in final form 10 Jun 2019.

\begin{abstract}
Background. Given the importance of English a global language and one of the four official languages used in FIFA, it is essential to acquaint students who are specialised in Physical Education with English sport terminology. Objectives. This study aims to determine the knowledge level of physical education students in Saudi universities' faculties and departments in relation to English sports terms. A survey was used to collect the relevant data from the participants. Methods. The study sample consisted of 600 students, who were selected using stratified random sampling during the academic year 2013-2014. The results indicate that the average percentage of correct answers was only $8.58 \%$. Results. The results also suggest that there are statistically differences for study variables related to students' performance. Those students who actively play sports performed better than those who do not. Moreover, those students who have at least one non-Arab parent performed better than students who are of Arab parents. The researchers also found that graduates of international schools outperformed their private and public school peers. Finally, fourth year students achieved higher results than the more junior ones. Conclusion. The paper concludes with recommendations to review our language policy in general and to consider the addition of a unit or a chapter specific to English sports terminology in the curriculum to be taught to students in lectures. The researchers also recommend using and developing this test as a standard to determine the level of students' proficiency and the extent of their knowledge of English sports terminology.
\end{abstract}

KEY WORDS: English for Specific Purposes, Sport Terms, English Terms, Knowledge of English Language, Physical Education Students

\section{INTRODUCTION}

Sport is a phenomenon which transcends the bounds of language and culture in today's world, since it is practiced internationally. Nevertheless, for a number of reasons, English has become the official language of sport and a way to communicate successfully with people from different cultures $(1,2)$. The gradual increase of the use of English sport terms in the world of sport reinforces the importance of familiarizing students of physical education and sports science with English sport terms. However, the prevalence of such terms does not only extend to the classroom or to the players of the respective sports. For instance, the increasing nature of prematch and post-match analyses as well as international sports conferences - whether they are on local, regional or international levels require grounded knowledge of the relevant English sports terminology. It is highly important that physical education departments in

*. Corresponding Author:

Abdel Rahman Mitib Altakhaineh, Assistant Professor

E-mail: abdelrahman.takhaine@aau.ac.ae 
universities provide both practical and theoretical knowledge with regard to sports and sports sciences, particularly with regard to English sports terminology. This is because English is one of the four official languages used in FIFA for international competitions (3). Thus, it is vital to equip students with both, theoretical and practical knowledge of sports and sports sciences, if higher educational institutions are to prepare students well enough for their future academic and professional lives.

This does not mean to say that one should give preference to the learning of foreign languages over the mother tongue - in this case, Arabic - but it is important to recognize the need to educate students to a highly proficient degree, allowing them to flourish both academically and professionally. Thus, when it comes to foreign language learning, it is not a case of preferring one language to another, rather it is a matter of preparing students so that they are not hindered by any linguistic deficiencies. The following section will highlight some previous studies carried out in a number of other languages, with regard to sport terminology.

\section{Literature Review}

Knowledge of English sport terminology is an important aspect of sport culture. Despite attempts to find equivalent synonyms of English sport term in various languages $(2,4,5)$, English sport loanwords have often survived in foreign languages (4). Živanović et al (2013) investigated term formation in Serbian with regard to sports terminology. They concluded that terminology is the 'most movable part of lexis and an evergrowing system' (5). Therefore, the Serbian language has seen an accelerated increase in the number of lexical items entering the lexis in the areas of physical education and sports terminology in recent years.

Milić (2013) also investigated sport terminology in Serbian. The author analysed the influence of English on Serbian sport terminology compiling a variety of dictionaries and glossaries to form a corpus. They concluded that there is a considerable influence of English on Serbian sport terminology, largely by the borrowing of English sport terms. Reinton (1978) also investigated the relationship between English sport terms and their Russian synonyms. Conducting this research at a time when the general attitude towards foreign language influence on the Russian language was viewed negatively (4), Reinton concluded that despite a considerable attempt to replace English loanwords with Russian synonyms. In addition to the aforementioned extralinguistic factor, linguistic factors also played a part in the Russian context, since English loanwords were viewed to be shorter, and thus easier to utter than their Russian counterparts. However, Reinton concluded that the attempt to replace English loanwords with Russian synonyms was not as successful as some might have thought at the time, given that in many cases, it is the English loanwords which have survived in the Russian language, thus rendering the Russian synonyms almost obsolete.

Given that sport has a way of transcending cultural and national bounds, it is no surprise that English sport terminology has found its way into the lexis of many languages, including Russian, Serbian, Arabic and many more. Knowledge of sport theories, concepts, apparatus, techniques, tactics and more have been developed over the centuries into a discipline to be studied academically in its own right. Physical education and sport sciences are not just a by-product of other disciplines, and should thus be treated and taught as an independent academic discipline with its own educational curriculum in Arab universities to students of this respective discipline (6). The motivation for this study stems from problems faced by students from the physical education and sports sciences department at Taibah University in Saudi Arabia, with regard to the use of sports terms in English as opposed to Arabic. Given that both researchers play active roles in academic teaching and guidance at the aforementioned department, they observed that students generally displayed a relatively weak level of proficiency with regard to English sports terms. Hence, after vaguely observing the low proficiency level of English sport terms of students in their department, the authors looked to measure their proficiency levels. However, after delving into the matter further, the researchers recognized a dearth of reliable and valid tools to measure students' proficiency of English sports terms at Taibah University.

Therefore, the current study aims to: (1) determine students' level of proficiency with regard to English sports terms in general, of students in physical education faculties and 
departments in the Saudi universities; (2) determine whether there are significant differences in results between athletic and nonathletic students; (3) determine whether there are significant differences in results according to the ethnicities of the students' parents; (4) determine whether there are significant differences in results according to the type of school the students attended; and finally (5) determine whether there are significant differences in results according to the students' year of study. The study seeks answers the following research questions:

- What is the total extent of students' knowledge of English sports terms in the departments of physical education faculties and departments in Saudi universities?

- Is there a significant difference between the responses of students who play sports and those who do not?

- Is there a significant difference between the responses of those students whose parents are of Arab origin as compared to those whose parents one or both- are not of Arab origin?

- Is there a significant difference between the responses of students who graduated from public, private and international schools, respectively?

- Is there a significant difference between the results of students, depending on their current year of study?

\section{MATERIALS AND METHODS}

The method of data collection used for this study was descriptive in nature, given that this type of data collection is more suitable to the nature and purpose of the study. Therefore, a survey was used to collect the relevant data.

Study Sample. The study sample consists of 600 students selected from the physical education departments of six universities in the Kingdom of Saudi Arabia. Students were selected from different years of study during the academic year 2013/2014, (Table 1).

Instrument. The researchers use different types of tests to analyze data collected for the purpose of this study. All items for total knowledge measurements were derived theoretically passed on several studies (6-10) (See Appendix No. 1).

The test designed by the researchers was reviewed by a number of academic teaching staff within the physical education department at Taibah University, as well as teaching staff in other universities. This was done in order to ensure that the test met high standards of reliability, validity and objectivity as possible. This test was then distributed to 40 students of the physical education and sports sciences department of Taibah University, as a pilot study. These study were then excluded from the study sample.

Validity of the Test. The test was appraised by a number of academic professors, and it was analysed for virtual validity. Following on from this, the 'easy', 'difficult' and discrimination coefficients were calculated in order to find the constructive validity. Table 2 displays results of the 'easy', 'discrimination', 'difficulty' and skewness coefficients for each question.

Test Reliability. The researchers used test application and reapplication on reliability sample which consisted of 40 students, who were then excluded from the study sample to find the reliability coefficient.

\section{RESULTS}

Table 4 indicates that the students of university 6 outperformed students from the other universities in that they recognized and translated the given sports phrases with greater accuracy, giving a mean of 11.12. The university that responded with the fewest number of correct answers was university 2 , with a mean of 6.94. The hierarchy of universities starting with the most accurate to the least accurate translations of the sports terms is as follows: university $6(\mathrm{M}=11.12)$, university $3(\mathrm{M}=8.87)$, university $5(\mathrm{M}=8.68)$, university $4(\mathrm{M}=8.19)$, university $1(\mathrm{M}=7.69)$, and university $2(\mathrm{M}=6.94)$.

The results in table 5 indicate that the students of university 6 once again outperformed their counterparts in recognizing the sport that the various English terms are most associated with. The hierarchy of correct answers for recognizing this task is identical to the translation task. The means of each university in order of correct answers is as follows: university $6(\mathrm{M}=11.37)$, university $3(\mathrm{M}=8.96)$, university $5(\mathrm{M}=8.65)$, university $4 \quad(\mathrm{M}=7.88)$, university $1(\mathrm{M}=7.73)$, and university $2(\mathrm{M}=6.87)$.

The results in tables 4 and 5 both suggest that the students of King Saud University displayed the highest levels of proficiency for translating English sports terms correctly, in addition to correctly recognizing the sport to which the terms are most commonly associated with. 
Table 1. Demographic Variables of the Study Sample

\begin{tabular}{|lcc|}
\hline Categories & No. of Students & $\%$ \\
Year of Study & 150 & 25.0 \\
$1^{\text {st }}$ & 150 & 25.0 \\
2nd & 150 & 25.0 \\
3rd & 150 & 25.0 \\
4th & 600 & 100 \\
Total & & \\
Students' Athletic Division & 401 & 66.8 \\
Non Student - Athlete & 199 & 33.2 \\
Student - Athlete & 600 & 100 \\
Total & & \\
Parents' ethnicity & 466 & 77.7 \\
Arab & 134 & 22.3 \\
Non-Arab & 600 & 100 \\
Total & & \\
School & 290 & 48.3 \\
Public & 171 & 28.5 \\
Private & 139 & 23.2 \\
International & 600 & 100 \\
Total & & \\
University & 100 & 16.7 \\
Faisal University & 100 & 16.7 \\
AlBahah University & 100 & 16.7 \\
King Abd Alaziz University & 100 & 16.7 \\
Um Alqura University & 100 & 16.7 \\
Taibah University & 100 & 16.7 \\
King Saud University & 600 & 100 \\
\hline Total & & \\
\hline
\end{tabular}

Table 2. Easy, difficulty and discrimination for each test question (Words \& Phrases)

\begin{tabular}{|c|c|c|c|}
\hline Question No. (Words \& Phrases) & Easy & Difficulty & Discrimination \\
\hline 1. & 0.63 & 0.37 & 0.88 \\
\hline 2. & 0.64 & 0.36 & 0.69 \\
\hline 3. & 0.96 & 0.31 & 0.75 \\
\hline 4. & 0.63 & 0.37 & 0.94 \\
\hline 5. & 0.43 & 0.57 & 0.88 \\
\hline 6. & 0.62 & 0.38 & 0.75 \\
\hline 7. & 0.70 & 0.30 & 0.63 \\
\hline 8. & 0.34 & 0.66 & 0.69 \\
\hline 9. & 0.39 & 0.61 & 0.88 \\
\hline 10. & 0.52 & 0.58 & 0.94 \\
\hline 11. & 0.65 & 0.35 & 0.69 \\
\hline 12. & 0.42 & 0.58 & 0.50 \\
\hline 13. & 0.68 & 0.32 & 0.88 \\
\hline 14. & 0.67 & 0.33 & 0.44 \\
\hline 15. & 0.64 & 0.36 & 0.50 \\
\hline 16. & 0.50 & 0.50 & 0.56 \\
\hline 17. & 0.59 & 0.41 & 0.63 \\
\hline 18. & 0.46 & 0.54 & 0.69 \\
\hline 19. & 0.37 & 0.63 & 0.50 \\
\hline 20. & 0.48 & 0.52 & 0.81 \\
\hline 21. & 0.57 & 0.43 & 0.37 \\
\hline 22. & 0.69 & 0.31 & 0.75 \\
\hline 23. & 0.58 & 0.42 & 0.88 \\
\hline 24. & 0.57 & 0.43 & 0.81 \\
\hline 25. & 0.32 & 0.68 & 0.31 \\
\hline 26. & 0.54 & 0.46 & 0.63 \\
\hline 27. & 0.38 & 0.62 & 0.63 \\
\hline 28. & 0.55 & 0.45 & 0.44 \\
\hline 29. & 0.61 & 0.39 & 0.69 \\
\hline 30. & 0.60 & 0.40 & 0.56 \\
\hline 31. & 0.67 & 0.33 & 0.31 \\
\hline 32. & 0.59 & 0.41 & 0.49 \\
\hline 33. & 0.51 & 0.49 & 0.38 \\
\hline 34. & 0.58 & 0.42 & 0.69 \\
\hline 35. & 0.37 & 0.67 & 0.69 \\
\hline 36. & 0.51 & 0.49 & 0.50 \\
\hline 37. & 0.58 & 0.42 & 0.81 \\
\hline 38. & 0.68 & 0.32 & 0.81 \\
\hline 39. & 0.67 & 0.33 & 0.75 \\
\hline 40. & 0.69 & 0.31 & 0.63 \\
\hline
\end{tabular}


Table 3. The mean, standard deviation and correlation factor between the first and second application

\begin{tabular}{|c|c|c|c|c|c|}
\hline \multirow[t]{2}{*}{ Question No. (Words \& Phrases) } & \multicolumn{2}{|c|}{ First application } & \multicolumn{2}{|c|}{ Second application } & \multirow[t]{2}{*}{ Correlation } \\
\hline & Mean & Standard deviation & Mean & Standard deviation & \\
\hline 1. & 0.68 & 0.39 & 0.51 & 0.21 & 0.802 \\
\hline 2. & 0.44 & 0.42 & 0.56 & 0.44 & 0.851 \\
\hline 3. & 0.64 & 0.42 & 0.68 & 0.35 & 0.689 \\
\hline 4. & 0.55 & 0.38 & 0.53 & 0.29 & 0.721 \\
\hline 5. & 0.66 & 0.44 & 0.60 & 0.21 & 0.882 \\
\hline 6. & 0.59 & 0.48 & 0.59 & 0.34 & 0.813 \\
\hline 7. & 0.54 & 0.43 & 0.43 & 0.17 & 0.890 \\
\hline 8. & 0.61 & 0.45 & 0.40 & 0.24 & 0.731 \\
\hline 9. & 0.60 & 0.43 & 0.39 & 0.22 & 0.788 \\
\hline 10. & 0.54 & 0.50 & 0.41 & 0.31 & 0.805 \\
\hline 11. & 0.44 & 0.52 & 0.44 & 0.14 & 0.797 \\
\hline 12. & 0.48 & 0.41 & 0.48 & 0.26 & 0.788 \\
\hline 13. & 0.70 & 0.35 & 0.66 & 0.34 & 0.815 \\
\hline 14. & 0.58 & 0.26 & 0.52 & 0.25 & 0.819 \\
\hline 15. & 0.56 & 0.24 & 0.47 & 0.29 & 0.910 \\
\hline 16. & 0.58 & 0.51 & 0.52 & 0.21 & 0.910 \\
\hline 17. & 0.49 & 0.28 & 0.53 & 0.22 & 0.852 \\
\hline 18. & 0.52 & 0.22 & 0.58 & 0.31 & 0.824 \\
\hline 19. & 0.56 & 0.25 & 0.67 & 0.25 & 0.875 \\
\hline 20. & 0.56 & 0.34 & 0.58 & 0.36 & 0.820 \\
\hline 21. & 0.42 & 0.22 & 0.52 & 0.34 & 0.855 \\
\hline 22. & 0.57 & 0.32 & 0.56 & 0.27 & 0.831 \\
\hline 23. & 0.49 & 0.21 & 0.64 & 0.38 & 0.841 \\
\hline 24. & 0.48 & 0.46 & 0.58 & 0.35 & 0.662 \\
\hline 25. & 0.51 & 0.17 & 0.55 & 0.36 & 0.830 \\
\hline 26. & 0.61 & 0.35 & 0.63 & 0.37 & 0.715 \\
\hline 27. & 0.62 & 0.26 & 0.50 & 0.36 & 0.759 \\
\hline 28. & 0.61 & 0.26 & 0.59 & 0.36 & 0.697 \\
\hline 29. & 0.85 & 0.45 & 0.54 & 0.31 & 0.844 \\
\hline 30. & 0.41 & 0.45 & 0.47 & 0.35 & 0.758 \\
\hline 31. & 0.39 & 0.37 & 0.44 & 0.44 & 0.744 \\
\hline 32. & 0.45 & 0.33 & 0.52 & 0.41 & 0.796 \\
\hline 33. & 0.38 & 0.36 & 0.66 & 0.25 & 0.827 \\
\hline 34. & 0.47 & 0.30 & 0.59 & 0.24 & 0.748 \\
\hline 35. & 0.51 & 0.35 & 0.42 & 0.35 & 0.799 \\
\hline 36. & 0.49 & 0.42 & 0.46 & 0.37 & 0.841 \\
\hline 37. & 0.52 & 0.52 & 0.55 & 0.32 & 0.82 \\
\hline 38. & 0.47 & 0.28 & 0.55 & 0.26 & 0.821 \\
\hline 39. & 0.48 & 0.39 & 0.46 & 0.28 & 0.889 \\
\hline 40. & 0.44 & 0.34 & 0.45 & 0.39 & 0.845 \\
\hline
\end{tabular}

The results from tables 4 and 5 show that the students of King Saud University performed better than students from the other five universities on both, the phrase translation and the sport association tasks. Based on the results, the faculty of sport science and physical practices is branched into many departments specialized in sport field in addition to the oldest institute for preparing physical education teachers merged with it. While students of Jazan's university gained the lowest score in the results of knowledge level, translation of physical terms in English correctly and know the meant game through the term due to the department was newly established in addition to it stopped working for some time.

According to the results mentioned in table 6 , the probability value of the phrase translation was $(0.000)$, and the probability value for sport association task was (0.000). These two values suggest highly significant differences in both the phrase translation and the sport association tasks, between students who are practicing athletes compared to those students who are not. Therefore, the results seem to indicate a strong positive correlation between students' performance on these two tasks and whether they are active sports athletes. This may be due to the fact that students who are active athletes practice different sports, which may require them to interact with other sportspersons and trainers, particularly if those sportspersons and trainers do not speak Arabic. The English language's current status as a lingua franca (11) suggests that English will most likely be that contact language. These results are compatible with the results of El-Saleh \& AlRahamna (2009), Albatikhy (2006) and Elhory (2003) $(12,13)$. 
Table 4. Means and standard deviations for the responses of translation of the sports phrases

\begin{tabular}{|c|c|c|c|c|c|c|c|c|c|c|c|c|}
\hline \multirow[t]{2}{*}{ Question (Words \& Phrases) } & \multicolumn{2}{|c|}{ University (1) } & \multicolumn{2}{|c|}{ University (2) } & \multicolumn{2}{|c|}{ University(3) } & \multicolumn{2}{|c|}{ University(4) } & \multicolumn{2}{|c|}{ University(5) } & \multicolumn{2}{|c|}{ University(6) } \\
\hline & M & SD & $\mathbf{M}$ & SD & M & SD & M & SD & M & SD & M & SD \\
\hline Athletic & 0.06 & 0.24 & 0.04 & 0.20 & 0.19 & 0.39 & 0.12 & 0.33 & 0.13 & 0.34 & 0.17 & 0.38 \\
\hline Droop shoot & 0.50 & 0.50 & 0.47 & 0.50 & 0.50 & 0.50 & 0.57 & 0.50 & 0.64 & 0.48 & 0.37 & 0.49 \\
\hline Field & 0.29 & 0.46 & 0.27 & 0.45 & 0.37 & 0.49 & 0.24 & 0.43 & 0.22 & 0.42 & 0.50 & 0.50 \\
\hline Crawl Stroke & 0.03 & 0.17 & 0.00 & 0.00 & 0.12 & 0.33 & 0.07 & 0.26 & 0.09 & 0.29 & 0.09 & 0.29 \\
\hline Ranking List & 0.06 & 0.24 & 0.02 & 0.14 & 0.02 & 0.14 & 0.04 & 0.20 & 0.00 & 0.00 & 0.06 & 0.24 \\
\hline Track & 0.45 & 0.50 & 0.42 & 0.50 & 0.62 & 0.49 & 0.46 & 0.50 & 0.43 & 0.50 & 0.74 & 0.44 \\
\hline Umpire & 0.16 & 0.37 & 0.16 & 0.37 & 0.16 & 0.37 & 0.29 & 0.46 & 0.20 & 0.40 & 0.34 & 0.48 \\
\hline Exercise & 0.26 & 0.44 & 0.25 & 0.44 & 0.32 & 0.47 & 0.11 & 0.31 & 0.13 & 0.34 & 0.34 & 0.48 \\
\hline Gymnastics & 0.16 & 0.37 & 0.16 & 0.37 & 0.19 & 0.39 & 0.25 & 0.44 & 0.27 & 0.45 & 0.33 & 0.47 \\
\hline Champ point & 0.14 & 0.35 & 0.15 & 0.36 & 0.19 & 0.39 & 0.26 & 0.44 & 0.13 & 0.34 & 0.31 & 0.46 \\
\hline Sprint & 0.18 & 0.39 & 0.16 & 0.37 & 0.21 & 0.41 & 0.30 & 0.46 & 0.29 & 0.46 & 0.35 & 0.48 \\
\hline Record & 0.03 & 0.17 & 0.07 & 0.26 & 0.14 & 0.35 & 0.20 & 0.40 & 0.12 & 0.33 & 0.21 & 0.41 \\
\hline Hurdle & 0.16 & 0.37 & 0.11 & 0.31 & 0.14 & 0.35 & 0.18 & 0.39 & 0.13 & 0.34 & 0.18 & 0.39 \\
\hline Balance & 0.14 & 0.35 & 0.14 & 0.35 & 0.12 & 0.33 & 0.20 & 0.40 & 0.12 & 0.33 & 0.28 & 0.45 \\
\hline Racket & 0.28 & 0.45 & 0.28 & 0.45 & 0.30 & 0.46 & 0.13 & 0.34 & 0.12 & 0.33 & 0.34 & 0.48 \\
\hline Triple-Jump & 0.19 & 0.39 & 0.15 & 0.36 & 0.29 & 0.46 & 0.37 & 0.49 & 0.40 & 0.49 & 0.46 & 0.50 \\
\hline Breast Stroke & 0.24 & 0.43 & 0.23 & 0.42 & 0.31 & 0.46 & 0.07 & 0.26 & 0.10 & 0.30 & 0.33 & 0.47 \\
\hline Performance & 0.18 & 0.39 & 0.14 & 0.35 & 0.29 & 0.46 & 0.24 & 0.43 & 0.33 & 0.47 & 0.41 & 0.49 \\
\hline Pole Vault & 0.26 & 0.44 & 0.27 & 0.45 & 0.33 & 0.47 & 0.19 & 0.39 & 0.22 & 0.42 & 0.48 & 0.50 \\
\hline Offence & 0.03 & 0.17 & 0.02 & 0.14 & 0.05 & 0.22 & 0.07 & 0.26 & 0.05 & 0.22 & 0.05 & 0.22 \\
\hline Athletic & 0.00 & 0.00 & 0.00 & 0.00 & 0.00 & 0.00 & 0.00 & 0.00 & 0.02 & 0.14 & 0.01 & 0.10 \\
\hline Droop shoot & 0.13 & 0.34 & 0.08 & 0.27 & 0.30 & 0.46 & 0.21 & 0.41 & 0.36 & 0.48 & 0.40 & 0.49 \\
\hline Field & 0.10 & 0.30 & 0.12 & 0.33 & 0.14 & 0.35 & 0.15 & 0.36 & 0.21 & 0.41 & 0.29 & 0.46 \\
\hline Crawl Stroke & 0.00 & 0.00 & 0.00 & 0.00 & 0.02 & 0.14 & 0.00 & 0.00 & 0.06 & 0.24 & 0.04 & 0.20 \\
\hline Ranking List & 0.16 & 0.37 & 0.13 & 0.34 & 0.13 & 0.34 & 0.23 & 0.42 & 0.22 & 0.42 & 0.31 & 0.46 \\
\hline Track & 0.14 & 0.35 & 0.15 & 0.36 & 0.17 & 0.38 & 0.22 & 0.42 & 0.13 & 0.34 & 0.28 & 0.45 \\
\hline Umpire & 0.10 & 0.30 & 0.10 & 0.30 & 0.14 & 0.35 & 0.16 & 0.37 & 0.24 & 0.43 & 0.30 & 0.46 \\
\hline Exercise & 0.27 & 0.45 & 0.30 & 0.46 & 0.33 & 0.47 & 0.20 & 0.40 & 0.10 & 0.30 & 0.45 & 0.50 \\
\hline Gymnastics & 0.08 & 0.27 & 0.05 & 0.22 & 0.09 & 0.29 & 0.07 & 0.26 & 0.12 & 0.33 & 0.17 & 0.38 \\
\hline Champ point & 0.14 & 0.35 & 0.12 & 0.33 & 0.12 & 0.33 & 0.14 & 0.35 & 0.14 & 0.35 & 0.24 & 0.43 \\
\hline Sprint & 0.05 & 0.22 & 0.05 & 0.22 & 0.04 & 0.20 & 0.05 & 0.22 & 0.07 & 0.26 & 0.11 & 0.31 \\
\hline Record & 0.11 & 0.31 & 0.09 & 0.29 & 0.22 & 0.42 & 0.22 & 0.42 & 0.34 & 0.48 & 0.40 & 0.49 \\
\hline Hurdle & 0.00 & 0.00 & 0.00 & 0.00 & 0.00 & 0.00 & 0.00 & 0.00 & 0.00 & 0.00 & 0.00 & 0.00 \\
\hline Balance & 0.26 & 0.44 & 0.26 & 0.44 & 0.30 & 0.46 & 0.10 & 0.30 & 0.10 & 0.30 & 0.36 & 0.48 \\
\hline Racket & 0.12 & 0.33 & 0.05 & 0.22 & 0.13 & 0.34 & 0.11 & 0.31 & 0.13 & 0.34 & 0.21 & 0.41 \\
\hline Triple-Jump & 0.61 & 0.49 & 0.56 & 0.50 & 0.51 & 0.50 & 0.39 & 0.49 & 0.41 & 0.49 & 0.38 & 0.49 \\
\hline Breast Stroke & 0.35 & 0.48 & 0.30 & 0.46 & 0.31 & 0.46 & 0.33 & 0.47 & 0.41 & 0.49 & 0.12 & 0.33 \\
\hline Performance & 0.46 & 0.50 & 0.40 & 0.49 & 0.36 & 0.48 & 0.48 & 0.50 & 0.53 & 0.50 & 0.29 & 0.46 \\
\hline Pole Vault & 0.35 & 0.48 & 0.30 & 0.46 & 0.29 & 0.46 & 0.33 & 0.47 & 0.43 & 0.50 & 0.12 & 0.33 \\
\hline Offence & 0.46 & 0.50 & 0.37 & 0.49 & 0.41 & 0.49 & 0.44 & 0.50 & 0.54 & 0.50 & 0.30 & 0.46 \\
\hline Total & 7.69 & 3.50 & 6.94 & 3.58 & 8.87 & 4.06 & 8.19 & 3.41 & 8.68 & 4.23 & 11.12 & 2.01 \\
\hline
\end{tabular}

Table 7 shows that the probability value of the phrase translation task was 0.000 , and the probability value for phrase game was 0.000 . These two values suggest significant differences in both the phrase translation and the sport association tasks between those students whose parents are of Arab ethnicity and those students whose parents are of non-Arab ethnicity. The latter performed significantly higher than the former. This result might be due to the increased exposure to English inside the home, if English is the student's mother tongue, or if English is used extensively as a language of communication between parents and their children, particularly from a young age (14).

Table 8 shows the results of a one-way ANOVA according to school sector. The probability value of the phrase translation task was 0.000 and the probability value for sport association task was 0.000 . Once again, these two values suggest significant differences in both tasks in relation to the different school sectors. A Scheffe post hoc test was used to identify where exactly the significant differences lie.
The results of the Scheffe post hoc test indicate a number of significant differences between the different school sectors. The first significant difference to be noted is between the results of students from public and private schools, given that students from private schools performed better. Moreover, students from international schools performed better than those from public schools. Finally, the mean of the correct responses from students of international schools was also greater than the mean of correct responses from students who had previously studied at private schools.

The aforementioned results of the Scheffe post hoc test might be attributed to the fact that international schools in Saudi Arabia follow certain curricula and methods of instruction, which are conducive to the learning and use of English sports terms. Moreover, the medium of instruction in international schools in Saudi Arabia is English $(14,15)$, thus encouraging the use of English and greater exposure to English vocabulary. These results are compatible with Aljarf (2004) and AlWazeer (2000), whose 
studies pointed to the ubiquity of English in international schools in Saudi Arabia. Not only do international schools use English as the medium of instruction, but the high-quality resources, effective teaching methods, appropriate testing techniques and up-to-date textbooks all contribute to effective teaching, and consequently, learning.

Table 5. Means and standard deviations for the questions of naming the game of the phrases

\begin{tabular}{|c|c|c|c|c|c|c|c|c|c|c|c|c|}
\hline \multirow[t]{2}{*}{ Question (Words \& Phrases) } & \multicolumn{2}{|c|}{ University (1) } & \multicolumn{2}{|c|}{ University (2) } & \multicolumn{2}{|c|}{ University (3) } & \multicolumn{2}{|c|}{ University (4) } & \multicolumn{2}{|c|}{ University (5) } & \multicolumn{2}{|c|}{ University (6) } \\
\hline & $\mathbf{M}$ & SD & $\mathbf{M}$ & SD & $\mathbf{M}$ & SD & $\mathbf{M}$ & SD & $\mathbf{M}$ & SD & M & SD \\
\hline Athletic & 0.15 & 0.36 & 0.16 & 0.37 & 0.18 & 0.39 & 0.05 & 0.22 & 0.21 & 0.41 & 0.19 & 0.40 \\
\hline Droop shoot & 0.65 & 0.48 & 0.53 & 0.50 & 0.40 & 0.49 & 0.46 & 0.50 & 0.52 & 0.50 & 0.36 & 0.48 \\
\hline Field & 0.19 & 0.39 & 0.35 & 0.48 & 0.46 & 0.50 & 0.27 & 0.45 & 0.24 & 0.43 & 0.51 & 0.50 \\
\hline Crawl Stroke & 0.02 & 0.14 & 0.09 & 0.29 & 0.10 & 0.30 & 0.00 & 0.00 & 0.15 & 0.36 & 0.11 & 0.31 \\
\hline Ranking List & 0.07 & 0.26 & 0.00 & 0.00 & 0.09 & 0.29 & 0.02 & 0.14 & 0.06 & 0.24 & 0.07 & 0.26 \\
\hline Track & 0.34 & 0.48 & 0.43 & 0.50 & 0.58 & 0.50 & 0.52 & 0.50 & 0.41 & 0.49 & 0.69 & 0.46 \\
\hline Umpire & 0.17 & 0.38 & 0.12 & 0.33 & 0.15 & 0.36 & 0.30 & 0.46 & 0.24 & 0.43 & 0.34 & 0.48 \\
\hline Exercise & 0.20 & 0.40 & 0.30 & 0.46 & 0.21 & 0.41 & 0.15 & 0.36 & 0.22 & 0.42 & 0.37 & 0.49 \\
\hline Gymnastics & 0.09 & 0.29 & 0.11 & 0.31 & 0.22 & 0.42 & 0.29 & 0.46 & 0.23 & 0.42 & 0.31 & 0.46 \\
\hline Champ point & 0.16 & 0.37 & 0.13 & 0.34 & 0.18 & 0.39 & 0.23 & 0.42 & 0.24 & 0.43 & 0.32 & 0.47 \\
\hline Sprint & 0.23 & 0.42 & 0.13 & 0.34 & 0.35 & 0.48 & 0.27 & 0.45 & 0.24 & 0.43 & 0.37 & 0.49 \\
\hline Record & 0.09 & 0.29 & 0.03 & 0.17 & 0.17 & 0.38 & 0.15 & 0.36 & 0.12 & 0.33 & 0.19 & 0.39 \\
\hline Hurdle & 0.15 & 0.36 & 0.09 & 0.29 & 0.12 & 0.33 & 0.20 & 0.40 & 0.06 & 0.24 & 0.15 & 0.36 \\
\hline Balance & 0.17 & 0.38 & 0.11 & 0.31 & 0.19 & 0.39 & 0.13 & 0.34 & 0.19 & 0.39 & 0.29 & 0.46 \\
\hline Racket & 0.30 & 0.46 & 0.26 & 0.44 & 0.32 & 0.47 & 0.18 & 0.39 & 0.11 & 0.31 & 0.37 & 0.49 \\
\hline Triple-Jump & 0.26 & 0.44 & 0.18 & 0.39 & 0.44 & 0.50 & 0.22 & 0.42 & 0.31 & 0.46 & 0.46 & 0.50 \\
\hline Breast Stroke & 0.14 & 0.35 & 0.21 & 0.41 & 0.18 & 0.39 & 0.18 & 0.39 & 0.16 & 0.37 & 0.32 & 0.47 \\
\hline Performance & 0.23 & 0.42 & 0.23 & 0.42 & 0.33 & 0.47 & 0.15 & 0.36 & 0.29 & 0.46 & 0.41 & 0.49 \\
\hline Pole Vault & 0.14 & 0.35 & 0.27 & 0.45 & 0.33 & 0.47 & 0.25 & 0.44 & 0.30 & 0.46 & 0.52 & 0.50 \\
\hline Offence & 0.00 & 0.00 & 0.03 & 0.17 & 0.08 & 0.27 & 0.07 & 0.26 & 0.16 & 0.37 & 0.08 & 0.27 \\
\hline Athletic & 0.00 & 0.00 & 0.00 & 0.00 & 0.05 & 0.22 & 0.00 & 0.00 & 0.03 & 0.17 & 0.03 & 0.17 \\
\hline Droop shoot & 0.13 & 0.34 & 0.18 & 0.39 & 0.29 & 0.46 & 0.20 & 0.40 & 0.24 & 0.43 & 0.38 & 0.49 \\
\hline Field & 0.16 & 0.37 & 0.07 & 0.26 & 0.02 & 0.14 & 0.21 & 0.41 & 0.19 & 0.39 & 0.26 & 0.44 \\
\hline Crawl Stroke & 0.00 & 0.00 & 0.03 & 0.17 & 0.10 & 0.30 & 0.03 & 0.17 & 0.09 & 0.29 & 0.09 & 0.29 \\
\hline Ranking List & 0.09 & 0.29 & 0.06 & 0.24 & 0.15 & 0.36 & 0.24 & 0.43 & 0.22 & 0.42 & 0.28 & 0.45 \\
\hline Track & 0.13 & 0.34 & 0.13 & 0.34 & 0.13 & 0.34 & 0.24 & 0.43 & 0.25 & 0.44 & 0.31 & 0.46 \\
\hline Umpire & 0.14 & 0.35 & 0.06 & 0.24 & 0.21 & 0.41 & 0.22 & 0.42 & 0.22 & 0.42 & 0.31 & 0.46 \\
\hline Exercise & 0.20 & 0.40 & 0.22 & 0.42 & 0.26 & 0.44 & 0.22 & 0.42 & 0.15 & 0.36 & 0.43 & 0.50 \\
\hline Gymnastics & 0.05 & 0.22 & 0.08 & 0.27 & 0.13 & 0.34 & 0.11 & 0.31 & 0.08 & 0.27 & 0.17 & 0.38 \\
\hline Champ point & 0.19 & 0.39 & 0.05 & 0.22 & 0.08 & 0.27 & 0.23 & 0.42 & 0.31 & 0.46 & 0.27 & 0.45 \\
\hline Sprint & 0.08 & 0.27 & 0.05 & 0.22 & 0.12 & 0.33 & 0.03 & 0.17 & 0.13 & 0.34 & 0.14 & 0.35 \\
\hline Record & 0.14 & 0.35 & 0.12 & 0.33 & 0.29 & 0.46 & 0.18 & 0.39 & 0.28 & 0.45 & 0.40 & 0.49 \\
\hline Hurdle & 0.02 & 0.14 & 0.00 & 0.00 & 0.02 & 0.14 & 0.03 & 0.17 & 0.03 & 0.17 & 0.04 & 0.20 \\
\hline Balance & 0.27 & 0.45 & 0.21 & 0.41 & 0.28 & 0.45 & 0.17 & 0.38 & 0.18 & 0.39 & 0.41 & 0.49 \\
\hline Racket & 0.11 & 0.31 & 0.08 & 0.27 & 0.21 & 0.41 & 0.05 & 0.22 & 0.18 & 0.39 & 0.24 & 0.43 \\
\hline Triple-Jump & 0.57 & 0.50 & 0.50 & 0.50 & 0.44 & 0.50 & 0.38 & 0.49 & 0.37 & 0.49 & 0.42 & 0.50 \\
\hline Breast Stroke & 0.38 & 0.49 & 0.29 & 0.46 & 0.23 & 0.42 & 0.22 & 0.42 & 0.30 & 0.46 & 0.12 & 0.33 \\
\hline Performance & 0.45 & 0.50 & 0.34 & 0.48 & 0.29 & 0.46 & 0.45 & 0.50 & 0.35 & 0.48 & 0.27 & 0.45 \\
\hline Pole Vault & 0.35 & 0.48 & 0.29 & 0.46 & 0.26 & 0.44 & 0.28 & 0.45 & 0.27 & 0.45 & 0.12 & 0.33 \\
\hline Offence & 0.52 & 0.50 & 0.35 & 0.48 & 0.32 & 0.47 & 0.30 & 0.46 & 0.33 & 0.47 & 0.25 & 0.44 \\
\hline Total & 7.73 & 3.72 & 6.87 & 3.60 & 8.96 & 4.59 & 7.88 & 3.78 & 8.65 & 5.03 & 11.37 & 2.51 \\
\hline
\end{tabular}

Table 6. T-test results for the cognitive knowledge in detecting the translation of the sports phrases for the students at selected the Saudi universities according to the Student's sport status

\begin{tabular}{|lcccccc|}
\hline Sport status & N & Mean & SD & t-value & P & Result \\
Phrase translation & & & & 11.43 & 0.000 & Sig \\
$\quad$ Non Student - Athlete & 401 & 7.46 & 3.08 & & & \\
$\quad$ Student - Athlete & 199 & 10.84 & 3.98 & & & \\
Phrase game & & & & 11.71 & 0.000 & Sig \\
$\quad$ Non Student - Athlete & 401 & 7.30 & 3.28 & & & \\
$\quad$ Student - Athlete & 199 & 11.14 & 4.63 & & & \\
\hline
\end{tabular}

Table 7. T-test results for the cognitive knowledge in detecting the translation of the sports phrases for the students at selected the Saudi universities according to the Student's Parents origins

\begin{tabular}{|lcccccc|}
\hline Parents origins & $\mathbf{N}$ & Mean & Sd & $\boldsymbol{t}$-value & $\mathbf{P}$ & Result \\
Phrase translation & & & & 15.54 & 0.000 & Sig \\
$\quad$ Arabic & 466 & 7.50 & 3.31 & & & \\
$\quad$ Non-Arabian & 134 & 12.34 & 2.63 & & & \\
Phrase game & & & & 16.06 & 0.000 & Sig \\
$\quad$ Arabic & 466 & 7.35 & 3.50 & & & \\
$\quad$ Non-Arabian & 134 & 12.86 & 3.51 & & & \\
\hline
\end{tabular}


Table 8. One-way ANOVA results for the cognitive knowledge in detecting the translation of the sports phrases for the students at selected Saudi universities according to the school sector

\begin{tabular}{|lcccccc|}
\hline $\begin{array}{l}\text { School Sector } \\
\text { Phrase translation }\end{array}$ & N & Mean & SD & $\boldsymbol{f}$ value & P & Result \\
Public & 290 & 6.43 & 2.73 & & & \\
Private & 171 & 9.61 & 3.10 & & & \\
International & 139 & 11.80 & 3.52 & & & \\
Phrase game & & & & 165.88 & 0.000 & Sig \\
Public & 290 & 6.21 & 2.90 & & & \\
Private & 171 & 9.53 & 3.30 & & & \\
International & 139 & 12.34 & 4.22 & & & \\
\hline
\end{tabular}

Table 9. Scheffe post hoc test for the cognitive knowledge in detecting the translation of the sports phrases for the students at selected Saudi universities according to the school sector

\begin{tabular}{|cccc|}
\hline Means & Category & Private & International \\
Phrase translation & & & $*$ \\
6.43 & Public & $*$ & $*$ \\
9.61 & Private & & \\
11.80 & International & & $*$ \\
Phrase game & & & $*$ \\
6.21 & Public & $*$ & \\
9.53 & Private & & \\
12.34 & International & & \\
\hline
\end{tabular}

(*) indicate significant differences between the two categories

Table 10. One-way ANOVA results for the cognitive knowledge in detecting the translation of the sports phrases for the students at selected Saudi universities according to the studying year level

\begin{tabular}{|lcccccc|}
\hline $\begin{array}{l}\text { Studying year level } \\
\text { Phrase translation }\end{array}$ & $\mathbf{N}$ & Mean & SD & f value & $\mathbf{P}$ & Result \\
1st & 150 & 6.19 & 3.22 & & & \\
2nd & 150 & 7.60 & 2.66 & & & \\
3rd & 150 & 8.46 & 3.06 & & & \\
4th & 150 & 12.08 & 3.29 & & & \\
Phrase game & & & & 106.40 & 0.000 & Sig \\
1st & 150 & 5.93 & 3.42 & & & \\
2nd & 150 & 7.45 & 2.85 & & & \\
3rd & 150 & 8.33 & 3.29 & & & \\
4th & 150 & 12.59 & 3.90 & & & \\
\hline
\end{tabular}

Table 11. Scheffe post hoc test for the cognitive knowledge in detecting the translation of the sports phrases for the students at selected Saudi universities according to the year of study

\begin{tabular}{|lcccc|}
\hline Means & Category & $\mathbf{2}^{\text {nd }}$ & $\mathbf{3}^{\text {rd }}$ & $\mathbf{4}^{\text {th }}$ \\
Phrase translation & & & & \\
6.19 & $1^{\text {st }}$ & $*$ & $*$ & $*$ \\
7.60 & $2^{\text {nd }}$ & & & $*$ \\
8.46 & $3^{\text {rd }}$ & & & $*$ \\
12.08 & $4^{\text {th }}$ & & & \\
Phrase game & & & & \\
5.93 & $1^{\text {st }}$ & $*$ & $*$ & $*$ \\
7.45 & $2^{\text {nd }}$ & & & $*$ \\
8.33 & $3^{\text {rd }}$ & & & $*$ \\
12.59 & $4^{\text {th }}$ & & & \\
\hline
\end{tabular}

(*) indicate significant differences between the two categories

Table 10 shows the results of a one-way ANOVA according to the students' year of study. The probability value of the phrase translation task was (0.000) and the probability value for linking the phrase with the sport it is most commonly associated with was (0.000). These two values suggest significant differences in both the phrase translation task and phrase association task in relation to the between among the school sector categories. Scheffe post hoc test was used to specify the differences sources in the studying year level variable. Results are included in the following table:

The results of the Scheffe post hoc test as mentioned in table 11 display where the significant differences lie in relation to the students' year of study. Students who were in their fourth year of study outperformed the 
students of earlier years of study on both tasks. In addition, there were significant differences between the results of students from earlier years of study and those students who were of subsequent years of study. Thus, the results show a strong positive correlation between the year of study and the number of correct answers for both tasks. The gradation of practical and theoretical knowledge as students' progress in their academic education is possibly a factor in the high performance of the fourth year students. Furthermore, exposure to training sessions and conferences where the more senior students have greater exposure to English sports terms could also suggest a higher proficiency level. Consequently, first year students do not have as much exposure as their senior peers. These results are in line with the results found by AlBtikhy (2006) and El-Saleh and AlRahmana (2009) (12, 16).Thus, one may conclude that as the student engages in the education process and receives more specialized courses, their knowledge of English sport terms, exposure, and experience of translating the terms increases significantly.

Table 12. Scheffe post hoc test for the cognitive knowledge in detecting the translation of the sports phrases for the students at selected Saudi universities according to the university

\begin{tabular}{|c|c|c|c|c|c|c|}
\hline Means & University & 2 & 3 & 4 & 5 & 6 \\
\hline \multicolumn{7}{|c|}{ Phrase translation } \\
\hline 7.69 & Faisal University & & & & & * \\
\hline 6.94 & Jazan University & & $*$ & & * & $*$ \\
\hline 8.87 & King Abd Alaziz University & & & & & * \\
\hline 8.19 & Um Alqura University & & & & & * \\
\hline 8.68 & Taibah University & & & & & $*$ \\
\hline 11.12 & King Saud University & & & & & \\
\hline \multicolumn{7}{|c|}{ Total \%8.58 } \\
\hline \multicolumn{7}{|c|}{ Phrase game } \\
\hline 7.73 & Faisal University & & & & & * \\
\hline 6.87 & Jazan University & & $*$ & & & * \\
\hline 8.96 & King Abd Alaziz University & & & & & * \\
\hline 7.88 & Um Alqura University & & & & & $*$ \\
\hline 8.65 & Taibah University & & & & & $*$ \\
\hline 11.37 & King Saud University & & & & & \\
\hline
\end{tabular}

(*) indicate significant differences between the two categories

The results of the Scehffe post hoc test, which was carried out to determine significant differences between the different universities, show that university 6 outperformed the other universities on both tasks. The results presented in the table 12 show that the differences were between:

- University 6 and the other universities in favor of university 6 as it recorded the greater mean for the phrase translation and the sport that the phrase refer to.

- University 2 and university 3 in favor of university 3 as it recorded the greater mean for the phrase translation and the sport that the phrase refers to.

- University 2 and university 5 in favor of university (5) as it recorded the greater mean for the phrase translation.

The researchers indicated to an important point in table 12 where the purpose of this study to know the general level of knowledge to universities as a whole not the order of universities. So, table 12 indicates to the level of knowledge for students in physical education faculties and departments in Saudi universities $(8.85 \%)$ which is very low percentage in translation of terms. knowing the physical game through the term was also very low where it reached to $(8.57 \%)$ after taking the mean of all universities. The researchers attribute this low level to absence of incentives, in generally, to learn English language and English terms. This is compatible with Steinberg's (2001) study in which weak self confidence in learning languages in addition to weak teaching in schools negatively affect student's level of English physical languages. Also neglect to put English terms in the courses and curricula or absence of these terms in addition to there is no obligation to the student to use these English terms in lectures or tests and weakness of family capability to develop their children English language and neglecting the education part of English to their children completely. This seems also compatible with AlHaj Essa and AlMetawa's (1988) and AlSebay's (1995) studies. 


\section{CONCLUSION}

In light of the previous results, the researchers conclude the following:

- The proficiency of students' in terms of English sport terms in the physical education faculties and departments in Saudi universities is, on the whole, very weak. Thus, the average percentage of correct answers is $8.58 \%$.

- Participants who were athletically active performed significantly better than those who are not.

- Students who have at least one parent of nonArab ethnicity performed significantly better than those students, both parents of whom, are of Arab ethnicity.

- Students who had studied at international schools performed significantly better than those who had studied at private and public schools, and students who had studied at private schools performed significantly better than those from public schools.

- Students who were in higher years of study performed significantly better than those students who were in earlier years of study.

- In light of the aforementioned conclusions, the researchers recommend the following:

- To reconsider our linguistic policy: this includes the development of strategies and plans to develop English proficiency and the inclusion of sports terminology in the curricula.

- To review our current curricula and teaching methods and to include English sport terms related to each curriculum for all courses are taught in Arabic.

- To reconsider our education policy: to get educational institutions (i.e. schools and universities) more involved in teaching English language and sport terminology.

- To append a unit or a chapter dedicated to English sports terms related to each curriculum in lectures and exams.

- To encourage more empirical studies to be carried out in other Arabic-speaking universities, in order to identify the challenges faced by students and to find a solution for them.

- To validate and, if need be, modify the instrument used in this paper as a valid and reliable measure of students' knowledge of English sports terms.

\section{APLICABLE REMARKS}

- Students at Saudi universities who major in physical education should improve their English language, in general, and English sport terminology, in particular.

- Educators and curriculum designers need to design English for specific purposes course, i.e. English for sports to be studied by students at Saudi universities.

- Saudi universities need to hire English language instructors who are knowledgeable in English sport terminology.

\section{REFERENCES}

1. Altakhaineh ARM, Khawlah M, AL-Tkhayneh H, Rahrouh N. The Effect of the Gender and Culture of the IELTS Examiner on the Examinees' Performance on the IELTS Speaking Test in the UAE Context. Int J Arabic English Stud. 2019;19(1):33-52. doi: 10.33806/ijaes2000.19.1.2

2. Milić M. The influence of English on Serbian sports terminology. ESP Today-J English Spec Purpose. 2013.

3. Translation blog. Language in Sports: International Communication at an Organizational Level: Translation blog; 2017 [cited 2019 Jan 21]. Available from: http://translationblog.trustedtranslations.com/language-sports-international-communicationorganizational-level2017-07-27.html.

4. Reinton JE. The relationship between english loanwords and their synonyms in Russian Sport terminology. Scando-Slavica. 2008;24(1):217-237. doi: 10.1080/00806767808600749

5. Živanović N, Ranđelović N, Milošević Z, Piršl D, Bogdanović N, Rančić S. Syntagmas of sports terminology in Serbian language. Fact Univ Series Phys Educ Sport. 2013;11(1):81-92.

6. Al-Kholy A. Sources of physical knowledge for students of Helwan university. Fourth scientific conference, physical education studies and researches; Helwan university, Egypt1983.

7. Al-Amir K. Estimation of the knowledge level for football trainers and administrative workers in Kuwait in the field of athletes' nutrition. Sci Mag Phys Sport Educ. 1997;12.

8. Al-Mohands AAE-K, Bakry SAA-H. Translation in King Saud university. Seminar about generalization of Arabic localization and development of translation in Saudi Arabia; KSA1998. 
9. Hashisho N. Developing Guidelines for Teaching English as a second language. Amman: AAUGS; 2005.

10. Ma'touq A. The language outcome its importance, sources and means to develop. Kuwait: Alam Almarefa serial, the national assembly for culture and arts in Kuwait; 1996.

11. Seidlhofer B. Research Perspectives on Teaching English as a Lingua Franca. Ann Rev Appl Ling. 2004;24. doi: 10.1017/s0267190504000145

12. Al-Batikhy N. Knowledge/Cognitive abilities of table tennis coaches in Jordan. Jordan2006.

13.El-Hory M. The scientific knowledge outcome for Karate trainers in Jordon. Jordon: El-Yarmouk University; 2003.

14. Al-Jarf RS. Attitudes of youth towards using of both Arabic and English languages in education. KSA: King Saud university; 2004.

15. Al-Wazeer A. The physical knowledge of physical education teachers in Madina region. 2000.

16.El-Saleh M, Al-Rahamna H. Measurement of knowledge level for physical education students in Jordan University in relation to physical fitness. Sixth scientific physical conference, sport and development: a future view for the third millennium2009.

\section{Appendix. (1)}

Dear student,

Peace upon you

The researchers perform a study titled:

Measuring the total knowledge of English Sports Terminology by the Physical Education Students at Saudi Universities

So, they ask students to respond on the test questions through writing translation to the term in place. These terms are related to sport games and there some words belong to more than one game.

The researchers ask you to respond objectively on this test questions where this information will be secret and used only for scientific purposes.

Sincerely,

The researchers

First: Please, fill in your personal data and shade choices:

\begin{tabular}{|llll|}
\hline Name: (as you like) & & \multicolumn{3}{c|}{ Study year } \\
Age: & & & First \\
Did you graduate in ......... School public school & Private & Foreign & Third fourth \\
(Student item) are you previous player? & Yes & No & Please shade the choice \\
(Parents' origin) is one of the parents a foreigner? & Yes & No & \\
\hline
\end{tabular}

Second: Please write translation of the term and in what game it is used?

\begin{tabular}{|c|c|c|c|c|c|c|c|}
\hline No. & Term & Translation & The game & No. & Term & Translation & The game \\
\hline 1 & Athletic & & & 21 & Cross over & & \\
\hline 2 & Droop shoot & & & 22 & Bench & & \\
\hline 3 & Field & & & 23 & Throw in & & \\
\hline 4 & Crawl Stroke & & & 24 & Turnover & & \\
\hline 5 & Ranking list & & & 25 & Block & & \\
\hline 6 & Track & & & 26 & Lop & & \\
\hline 7 & Umpire & & & 27 & Penetration & & \\
\hline 8 & Exercise & & & 28 & Zoon & & \\
\hline 9 & Gymnastic & & & 29 & Dribble & & \\
\hline 10 & Champ point & & & 30 & Penalties & & \\
\hline 11 & Sprint & & & 31 & Rotation & & \\
\hline 12 & Record & & & 32 & Serve & & \\
\hline 13 & Hurdle & & & 33 & Disqualification & & \\
\hline 14 & Balance & & & 34 & Fault & & \\
\hline 15 & Racket & & & 35 & Lot (Toss) & & \\
\hline 16 & Triple-jump & & & 36 & Fast break & & \\
\hline 17 & Breast stroke & & & 37 & Offside & & \\
\hline 18 & Performance & & & 38 & Feint & & \\
\hline 19 & Pole vault & & & 39 & Draw & & \\
\hline 20 & Offence & & & 40 & Referee & & \\
\hline
\end{tabular}

Thank you for your cooperation

Sincerely;

The researchers 